\title{
Ciudades biodiversas: mamíferos medianos de la Reserva Forestal Protectora Bosque Oriental de Bogotá D. C., Colombia
}

\section{J. Sebastián Jiménez-Alvarado \\ Catalina Moreno-Díaz}

Proyecto de Conservación de Aguas y Tierras, ProCAT Colombia/Internacional, Carrera 13 No. 96-82 Oficina 205, Bogotá, Colombia. jsjimenezalvarado@gmail.com

Andrés Felipe Alfonso

Anthony Giordano

Field Projects and Conservation Science, S.P.E.C.I.E.S., Ventura, CA, 93003, U.S.A.

\section{Mauricio Vela-Vargas}

School of Natural Resources and the Environment. University of Arizona. Tucson, AZ, U.S.A.

Proyecto de Conservación de Aguas y Tierras, ProCAT Colombia/Internacional, Carrera 13 No. 96-82 Oficina 205, Bogotá, Colombia

\section{Diego A. Gómez-Hoyos \\ José F. González-Maya}

Proyecto de Conservación de Aguas y Tierras, ProCAT Colombia/Internacional, Carrera 13 No. 96-82 Oficina 205, Bogotá,

Colombia

Los bosques de niebla representan el $0,3 \%$ de la superficie terrestre y presentan una alta diversidad de fauna y flora, la cual provee funciones importantes para la dinámica de los ecosistemas (Armenteras et al., 2007). Estos bosques, junto al ecosistema de páramo, son los principales reguladores y abastecedores de agua de las principales ciudades de Colombia (Armenteras et al., 2007). A pesar del avance de la frontera agrícola y ganadera hacia estas coberturas (Cabrera et al., 2011), y la considerable expansión de la mancha urbana en la región Andina, aún se mantienen relictos de bosque que albergan considerables elementos de la biodiversidad en áreas periurbanas de las grandes ciudades del país (Armenteras et al., 2003).

La Sabana de Bogotá ha enfrentado históricamente factores de amenaza como la ganadería y la agricultura extensiva, así como la expansión urbana (Casas, 1989). Estas amenazas han afectado la riqueza y composición de la biodiversidad, y en especial de los mamíferos de estas áreas, restringiendo su distribución local a frágiles remanentes de bosque dispersos en la zona urbana y sus alrededores (CAR \& CI, 2009). En este contexto de paisaje, los Cerros Orientales de Bogotá representan uno de los últimos remanentes de cobertura natural en las zonas circundantes de Bogotá, siendo fundamental tanto para conectar dos de las áreas de mayor importancia del país (i.e., Chingaza y Sumapáz), como para la provisión de servicios para la ciudad y la Sabana en general (CAR \& CI, 2009). En este sentido, generar herramientas científicas e información para su adecuada planificación es una oportunidad única para aportar a la integridad ecológica de la ciudad y la calidad de vida de sus habitantes. En esta contribución, presentamos un listado actualizado de los mamíferos medianos presentes en la Reserva Forestal Protectora Bosque Oriental de Bogotá.

El área de estudio comprende la Reserva Forestal Protectora Bosque Oriental de Bogotá que se localiza al oriente de la ciudad de Bogotá D.C., y comúnmente conocida como Cerros Orientales (Figura 1). La reserva cuenta con un área aproximada de 14.000 ha y un rango altitudinal entre los 2.575 y los $3.575 \mathrm{msnm}$ (CAR \& CI, 2009). Nuestro estudio se realizó específicamente en el borde Norte de la Reserva, en los remanentes de bosque asociados a diferentes conjuntos residenciales: Floresta de la Sabana $(4.81379 \mathrm{~N},-74.02578 \mathrm{~W})$, Bosque de Torca $(4.81906 \mathrm{~N},-74.02468 \mathrm{~W})$ y Portal de Fusca (4.82984N, $74.01627 \mathrm{~W})$, así como la finca MiraValle (4.79658N, -74.01099W). Estos remanentes de bosque hacen parte del sistema orográfico y de la estructura ecológica principal del distrito capital (CAR \& CI 2009).

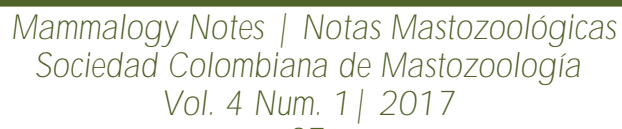


primera vez en zonas peri urbanas de la ciudad de Bogotá (González-Maya et al., 2017), y se suma al ensamble de carnívoros que están presentes en estas áreas. La presencia de roedores dispersores de semillas como la Guagua de Montaña (Cuniculus taczanowskii) y la Ardilla de Cola Roja (Notosciurus granatensis) fueron notables durante todo el muestreo, al igual que especies generalistas como la zarigüeya (Didelphis pernigra; Aranda, 2000).
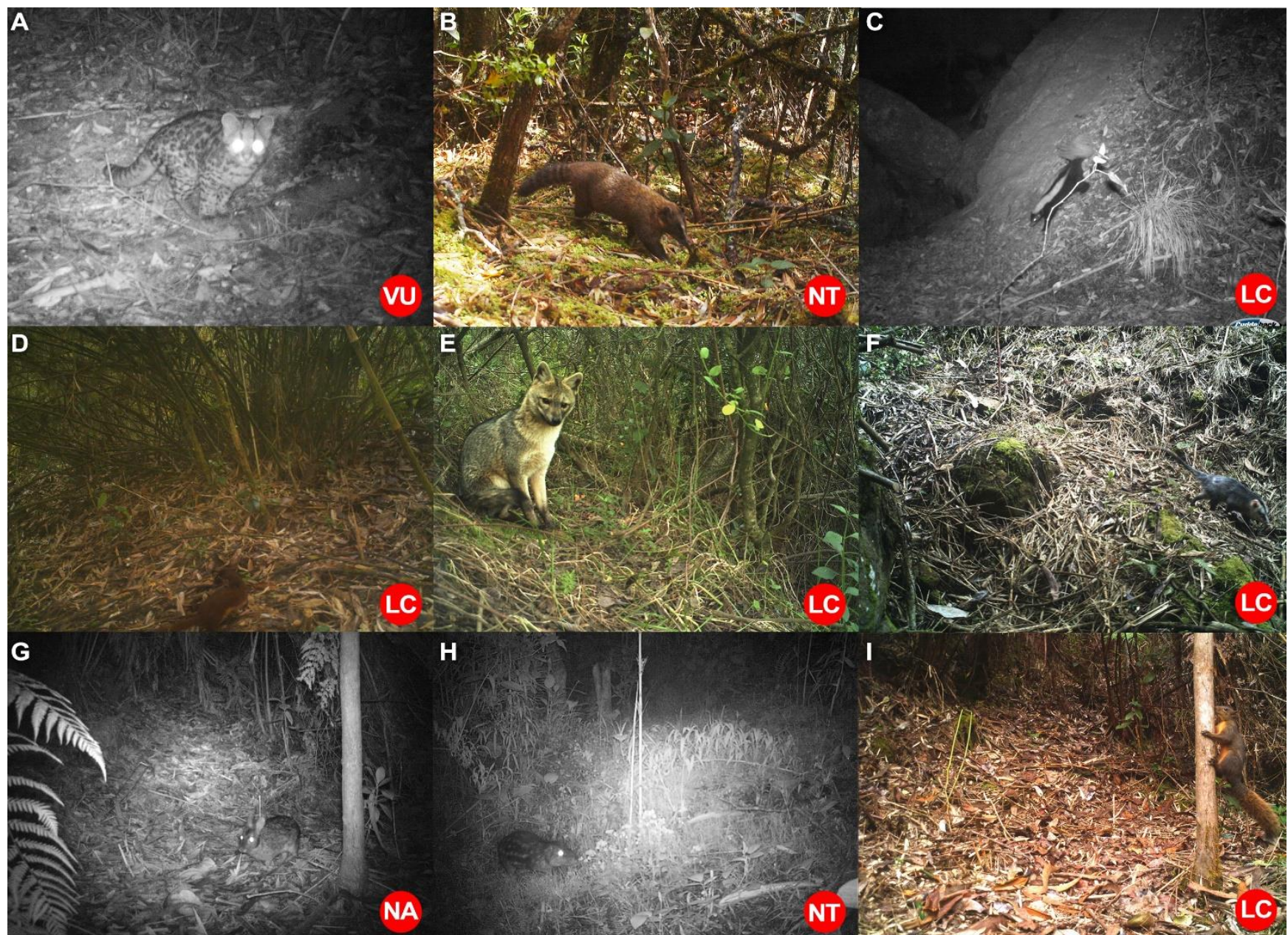

E
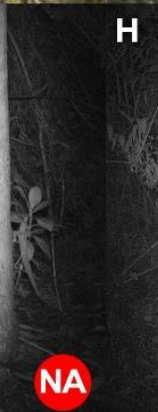

H

Figura 2. Especies de mamíferos medianos registrados en la porción norte de la Reserva Forestal Protectora Bosque Oriental de Bogotá,

Colombia. (A) Leopardus tigrinus, (B) Nasuella olivacea, (C) Conepatus semistriatus, (D) Mustela frenata, (E) Cerdocyon thous, (F)

Didelphis pernigra, (G) Sylvilagus andinus, (H) Cuniculus taczanowskii y (I) Notosciurus granatensis. Los círculos rojos indican el estado de conservación de acuerdo a la Lista Roja de Especies Amenazadas de UICN (2016): NA: No evaluado, LC: Baja preocupación,

NT: Casi amenazad y VU: Vulnerable.

Hasta la fecha no se había realizado un inventario sistemático de los mamíferos medianos para esta área en particular, y la única información disponible no presenta un listado de mamíferos específico a la reserva ni basado en esfuerzos sistemáticos de campo (sólo realizado para mamíferos pequeños y voladores), y se ha obtenido de manera global tomando como base la presencia de especies que se distribuyen cerca a esta área (CAR \& CI, 2009). Los esfuerzos sistemáticos de evaluación y monitoreo de la biodiversidad son fundamentales para su adecuado manejo adaptativo y la medición y aseguramiento del éxito en la gestión de estas áreas naturales. 


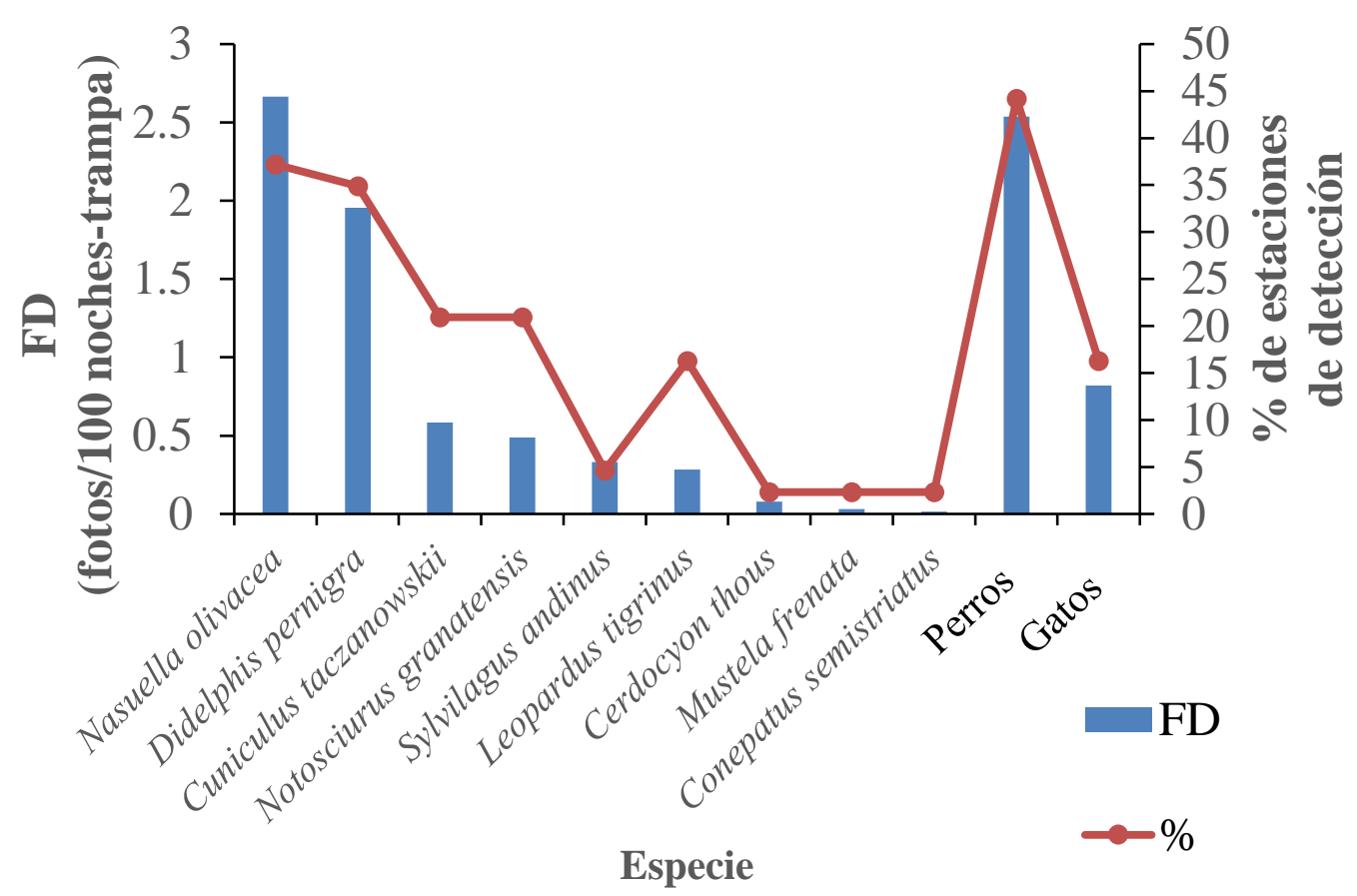

Figura 3. Distribución de la frecuencia de detección (FD) y porcentaje de estaciones (\%) de detección de mamíferos medianos en la porción norte de la Reserva Forestal Protectora Bosque Oriental de Bogotá, Colombia.

A pesar de que estas áreas se encuentran en alto grado de conservación y albergan especies de gran valor ecológico, se observa con preocupación una alta frecuencia de perros y gatos, tanto domésticos como ferales, en las zonas de bosque. Es importante resaltar la necesidad urgente de mejorar el manejo de fauna doméstica en áreas adyacentes y al interior de la Reserva, así como de políticas públicas claras desde la administración distrital y ambiental, para el manejo de la problemática y la reducción de la invasión y alarmante presencia y uso por individuos ferales y semiferales (Lessa et al., 2016; González-Maya et al., 2017). Esta problemática asociada al enorme riesgo que representa para las poblaciones silvestres, por efectos relacionados con la transmisión de enfermedades zoonóticas, la depredación, el desplazamiento competitivo, entre otros, que además representa un potencial problema de salud pública (Loss et al., 2013; Zapata-Ríos \& Branch, 2016).

La riqueza de mamíferos encontrados para la Reserva resalta su importancia como remanente de ecosistemas naturales fundamental para el mantenimiento de la estructura ecológica principal de la ciudad (Andrade \& Van der Hammen, 2003). A pesar de que múltiples amenazas continúan afectando la Reserva, desde la pérdida de cobertura por extracción de canteras, invasión de predios e incluso minería ilegal, hasta la cacería y la presencia de especies ferales y semiferales (Casas, 1989; Etter \& van Wyngaarden, 2000; CAR \& CI, 2009; González-Maya et al., 2017), la Reserva aún mantiene una composición significativa del ensamblaje de mamíferos medianos que se podrían esperar para la misma (CAR \& CI, 2009; Liévano-Latorre \& López Arévalo, 2014). Históricamente, los Cerros Orientales, también considerados cerros tutelares de la ciudad, han recibido poca atención de investigación e incluso de apropiación por parte de la capital, por lo que el rescate de la importancia y valor para la ciudad son claves tanto por conservar los remanentes de ecosistemas naturales como el patrimonio y calidad de vida de sus habitantes.

\section{Agradecimientos}

El presente trabajo es parte del proyecto Bogotá Biodiversa, liderado por ProCAT Colombia, con el apoyo de los conjuntos residenciales Portal de Fusca, Bosques de Torca y Floresta de la Sabana, S.P.E.C.I.E.S., UNAM y LEF-Pontificia Universidad Javeriana. Un especial agradecimiento a M. González, A. Uribe, I. Aragón, M. Giraldo, E. Cabo, M. Millán, J. Bobilla, A. Ruíz, M. Ruíz, G. Quiroga, A. Esparza, C.E. Aguilar, R.D. Herrera y A. Sánchez.

\section{Mammalogy Notes | Notas Mastozoológicas \\ Sociedad Colombiana de Mastozoología \\ Vol. 4 N um. 1| 2017}




\section{Referencias}

ANDRADE, G. \& VAN DER HAMMEN, T. 2003. Estructura ecológica principal de Colombia: primera aproximación. Ministerio de Ambiente, Vivienda y Desarrollo Territorial - MAVDT, Instituto de Hidrología, Meteorología y Estudios Ambientales - IDEAM, Bogotá, Colombia.

ARANDA, M. 2000. Huellas y otros rastros de los mamíferos grandes y medianos de México. Instituto de Ecología, A.C. Xalapa, México. 212 pp.

ARMENTERAS, D. et al. 2003. Andean Forest fragmentation and the representativeness of protected natural areas in the eastern Andes, Colombia. Biological Conservation 133: 245-256.

ARMENTERAS, D. et al. 2007. Evaluación del estado de los bosques de niebla y de la meta 2010 en Colombia. Instituto de Investigación de Recursos Biológicos Alexander Von Humboldt. Bogotá DC, Colombia, 72 pp.

CABRERA, E., et al. 2011. Memoria Técnica: Cuantificación de la tasa de Deforestación para Colombia, Periodo 1990-2000, 2000-2005. Instituto de Hidrología, Meteorología y Estudios Ambientales -IDEAM-. Bogotá D.C., Colombia. 22p.

CAR \& CI. 2009. Reserva Forestal Protectora Bosque Oriental de Bogotá: Inventario de Fauna. Corporación Autónoma Regional de Cundinamarca - CAR, Conservación Internacional Colombia, Bogotá, Colombia. 133pp

CASAS, F. 1989. The risks of environmental degradation in Bogotá, Colombia. Environemntal and Urbanization 1: 16-21.

ETTER, A. \& VAN WYNGAARDEN, W. 2000. Patterns of Landscape Transformation in Colombia, with Emphasis in the Andean Region. Ambio 29: 432439.

GONZÁLEZ-MAYA, J.F. et al. 2017. First confirmed record of the Striped Hog-nosed Skunk Conepatus semistriatus from peri-urban Bogotá, Colombia. Small Carnivore Conservation 55: 91-96.

IUCN Red List of Threatened Species. 2016. 〈www.iucnredlist.org>. Downloaded on 19 April 2017.

LESSA, I., CORRÊA SEABRA GUIMARÃES, T., DE GODOY BERGALLO, H., CUNHA, A. \& M. VIEIRA, E. 2016. Domestic dogs in protected areas: a threat to Brazilian mammals? Natureza \& Conservação 14: 46-56.

LOSS, S.R., WILL, T. \& MARRA, P.P. 2013. The impact of free-ranging domestic cats on wildlife of the United States. Nature Communications 4: 1396.

PAYÁN, E. \& GONZÁLEZ-MAYA, J.F. Distribución geográfica de la Oncilla (Leopardus tigrinus) en Colombia e implicaciones para su conservación. Revista Latinoamericana de Conservación 2(1): 51-59

PAYAN, E. \& DE OLIVEIRA, T. 2016. Leopardus tigrinus. The IUCN Red List of Threatened Species 2016: e.T54012637A50653881. http://dx.doi.org/10.2305/IUCN.UK.2016- LTS.T54012637A50653881.en..

ZAPATA-RÍOS, G. \& BRANCH, L.C. 2016. Altered activity patterns and reduced abundance of native mammals in sites with feral dogs in the high Andes. Biological Conservation 193: 9-16. 